Quantum interference and multielectron effects in highharmonic spectra of polar molecules

\author{
Journal Article \\ Author(s): \\ Rupenyan-Vasileva, Alisa Bohos (D); Kraus, Patrick M.; Schneider, Julian; Wörner, Hans Jakob \\ Publication date: \\ 2013-03 \\ Permanent link: \\ https://doi.org/10.3929/ethz-a-010780723
}

Rights / license:

In Copyright - Non-Commercial Use Permitted

Originally published in:

Physical Review A 87(3), https://doi.org/10.1103/PhysRevA.87.031401 


\title{
Quantum interference and multielectron effects in high-harmonic spectra of polar molecules
}

\author{
A. Rupenyan, P. M. Kraus, J. Schneider, and H. J. Wörner* \\ Laboratorium für Physikalische Chemie, ETH Zürich, \\ Wolfgang-Pauli-Strasse 10, 8093 Zürich, Switzerland
}

(Dated: February 21, 2013)

\begin{abstract}
We experimentally and theoretically analyze the manifestations of quantum interference and multiple ionization channels (multiple orbitals) in high-harmonic spectra of aligned $\mathrm{N}_{2} \mathrm{O}$ molecules. Increasing the probe wavelength from $1.17 \mu \mathrm{m}$ to $1.46 \mu \mathrm{m}$, we demonstrate the gradual disappearance of multi-electron effects and quantitatively explain the observation through calculations. We thus identify a minimum in the high-harmonic spectrum of $\mathrm{N}_{2} \mathrm{O}$ caused only by its structure. By comparing its position with that measured in the isoelectronic $\mathrm{CO}_{2}$ molecule for similar axis distributions, we find a difference of $10 \mathrm{eV}$, confirmed by ab initio quantum scattering calculations. Quantum interference in photorecombination is thus shown to be sensitive to subtle differences in the valence orbital structure of molecules with nearly identical lengths. This property may find applications in time-resolved studies.
\end{abstract}

PACS numbers: $33.20 . \mathrm{Xx}, 42.65 . \mathrm{Ky}, 42.50 . \mathrm{Hz}$

*woerner@phys. chem.ethz.ch; www.atto.ethz.ch 
High-harmonic spectroscopy (HHS) provides a new approach to investigating electronic structure and dynamics on femtosecond to attosecond time scales. In spite of important breakthroughs, seemingly simple molecules like $\mathrm{CO}_{2}$ and $\mathrm{N}_{2}$ are still the object of debates [1-11]. A minimum observed in spectra of aligned $\mathrm{CO}_{2}$ has first been interpreted in terms of quantum interference between the recombining photoelectron and the two-center structure of the highest-occupied molecular orbital (HOMO) $[2,3,10,11]$. However, a molecule exposed to a strong laser field can be ionized to several electronic states of the cation [6, 12-15], opening multiple channels for high-harmonic emission. Accordingly, the minimum in $\mathrm{CO}_{2}$ has later been attributed to electromagnetic interference between high-harmonic emission from multiple ionization channels of the molecule $[6,8,16]$. Using laser pulses with longer wavelengths $(1.45-1.7 \mu \mathrm{m})$, new evidence for a structural origin of the minimum has been obtained [9], in apparent contrast with previous results obtained in the wavelength range of 0.8-1.3 $\mu \mathrm{m}[6,8,16]$. Progress in high-harmonic spectroscopy will rely on achieving a better understanding of the sensitivities and mechanisms of the method.

In this Rapid Communication, we study the roles of quantum interference and multiple ionization channels in HHS of aligned weakly polar $\mathrm{N}_{2} \mathrm{O}$ molecules that we compare with the apolar isoelectronic $\mathrm{CO}_{2}$ molecules. We hereby address two questions: 1) what are the conditions under which quantum interference is observed? and 2) what is the relative role of the nuclear geometry and the electronic structure in defining the position of the associated spectral minima?

We answer the first question by studying high-harmonic spectra of $\mathrm{N}_{2} \mathrm{O}$ molecules over a range of intensities and wavelengths $(1.17-1.46 \mu \mathrm{m})$. We show that the position of the previously observed minimum in $\mathrm{N}_{2} \mathrm{O}$ [17] is intensity-dependent at $1.17 \mu \mathrm{m}$, owing to the interference of emission from multiple ionization channels, and that these multielectron effects progressively disappear with increasing wavelength. We quantitatively explain this observation through calculations $[6,18]$, that we extend to polar molecules for the first time. Recently, the phenomenon of quantum interference in polar molecules has been studied theoretically [19-21], but the role of multiple ionization channels has not been considered yet.

We answer the second question by comparing the purely structural signatures of two isoelectronic molecules with nearly identical length $\left(2.31 \AA\right.$ in $\mathrm{N}_{2} \mathrm{O}$ vs. $2.32 \AA$ in $\mathrm{CO}_{2}$ [22]) but different orbital shapes. In the scattering-wave description of photorecombination 
$[18,25]$ both the structure of the orbital and that of the scattering potential play a role in determining the positions of quantum interference minima, implying a sensitivity to both the electronic and the nuclear structure. In the present study, we find that the different minimum positions in $\mathrm{N}_{2} \mathrm{O}$ and $\mathrm{CO}_{2}$ reveal subtle differences of their HOMOs although the molecules have nearly identical lengths. We thus conclude that the electronic structure of $\mathrm{N}_{2} \mathrm{O}$ and $\mathrm{CO}_{2}$ dominates in determining the position of the structural minima. The results obtained by comparing two isoelectronic molecule over a wide range of experimental conditions thus allow us to disentangle the roles of the geometric structure, the electronic structure and multielectron effects which might be useful for time-resolved applications [15, 26-28].

The experimental setup consists of a titanium:sapphire laser system, an optical parametric amplifier (Light Conversion, TOPAS-HE) and a vacuum chamber. The laser system (8 mJ, $28 \mathrm{fs}, 1 \mathrm{kHz}$ ) provides pulses centered at $0.8 \mu \mathrm{m}$ which traverse an 80:20 beam splitter. The more intense beam is used to pump the TOPAS-HE that generates tunable infrared (IR) pulses in the range 1.1-1.5 $\mu \mathrm{m}$ with measured pulse durations of 40-50 fs. The minor part of the output is stretched to $120 \mathrm{fs}$ and used for non-adiabatic alignment of the molecules. The mid-IR probe beam is aligned parallel to the $0.8 \mu \mathrm{m}$ pump beam with a vertical offset of 0.7 $\mathrm{cm}$ and the two beams are focused into a gas jet inside a vacuum chamber using a $f=50$ $\mathrm{cm}$ spherical mirror. The molecular beam is generated by supersonic expansion through a pulsed valve with a $250 \mu \mathrm{m}$ orifice and a backing pressure of 5 bars. The focused laser beams intersect the gas jet 2-3 $\mathrm{mm}$ downstream of the nozzle. The high harmonics generated by the probe beam propagate into a spectrometer consisting of a $120 \mu \mathrm{m}$ wide entrance slit, a concave aberration-corrected grating (Shimadzu, 30-002) and an extended-dynamic-range microchannel-plate detector backed with a phosphor screen (Photonis, 37258). In the present study, we have not observed orientation under the same experimental conditions use to orient OCS [29] which we attribute to the weak polarity of $\mathrm{N}_{2} \mathrm{O}$.

Figure 1 shows high-harmonic spectra of $\mathrm{N}_{2} \mathrm{O}$ molecules aligned parallel to the polarization direction of the probe beam for the three wavelengths $1.17 \mu \mathrm{m}(\mathrm{a}), 1.36 \mu \mathrm{m}$ (b) and $1.46 \mu \mathrm{m}$ (c) and several intensities given in the legends. The molecules were impulsively aligned using a $120 \mathrm{fs}, 0.8 \mu \mathrm{m}$ pulse and were recorded at the first half-revival of the rotational dynamics. All spectra show distinct minima in the range of 50-55 eV. At the shortest wavelength, the minimum position changes with the intensity of the probe pulse, moving from $50 \mathrm{eV}$ to $55 \mathrm{eV}$. At $1.36 \mu \mathrm{m}$, the position of the minimum moves by less than $2 \mathrm{eV}$ 
and at $1.46 \mu \mathrm{m}$ the shift is on the order of $1 \mathrm{eV}$. We thus observe a progressive decrease of the intensity-dependence of the minimum in $\mathrm{N}_{2} \mathrm{O}$ with increasing wavelength of the probe pulse for similar relative variations of the probe intensity. This situation is reminiscent of $\mathrm{CO}_{2}$ where such a result was however obtained in different studies using very different laser sources $[8,9,16]$. Here, we show that the intensity dependence of the minimum position disappears toward longer wavelengths even when pulses consisting of 7-9 cycles are used. We show that the same is true for $\mathrm{CO}_{2}$ molecules in a companion paper [30].

We now discuss our theoretical model (details are given in Ref. [30]). The valence electronic structure of $\mathrm{N}_{2} \mathrm{O}$ consists of the orbitals with the following symmetries and binding energies (equal to the vertical ionization energies within Koopmans' theorem): $\pi, 12.89 \mathrm{eV}$, $\sigma, 16.38 \mathrm{eV}, \pi, \sim 18.2 \mathrm{eV}, \sigma, 20.11 \mathrm{eV}$ [31]. Both ionization from and recombination to HOMO are suppressed when the molecule is aligned parallel to the generating laser field. Therefore, the contribution of HOMO-1 becomes significant and is fully included in our model. Lower-lying orbitals (HOMO-2 and HOMO-3) were found to contribute insignificantly and were not included in the calculations.

We use a velocity-gauge model for strong-field ionization $[6,32,33]$ and extend it to take into account the polarity of $\mathrm{N}_{2} \mathrm{O}$ and the Stark effect. Our model thus incorporates the directional dependence of the effective binding energy resulting from the Stark effect, as e.g. in Ref. [34]. The polarization of the orbitals which becomes important in systems with large polarizabilities [35] is not included here. The orbitals were obtained from a HartreeFock calculation using a correlation-consistent polarized valence-triple-zeta (cc-pVTZ) basis set and are shown in Fig. 2a. The permanent dipole moments and polarizabilities were obtained by fitting a second-order polynomial to the orbital binding energies obtained from a MP2 calculation with an applied static field in the range of 0-0.1 atomic units. The resulting tunnel ionization rates for the $\mathrm{HOMO}$ and $\mathrm{HOMO}-1$ of $\mathrm{N}_{2} \mathrm{O}$ are shown in Fig. $2 \mathrm{~b}$. Whereas the ionization rate of HOMO is essentially inversion symmetric, the ionization rate of HOMO-1 is strongly asymmetric, with a maximum amplitude for removing the electron via the oxygen atom. This asymmetry is dominated by the permanent dipole moment of the orbital, in analogy to OCS [36].

We use the strong-field approximation [37] to calculate channel-specific continuum electron wave packets. These wave packets contain all laser-pulse specific properties of highharmonic emission. In particular, the relative phase contributed by the propagation step as 
a consequence of different ionization potentials $\left(I_{p}\right)$, which is sometimes approximated by $\Delta I_{p} \tau$ with $\tau$ the transit time of the electron [8,38,39], is naturally included in the spectral phase of the continuum electron wave packets. We model photorecombination using ab initio quantum scattering calculations $[40,41]$ based on the Hartree-Fock orbitals described above. The calculated photoionization cross sections are shown in Fig. 2c. Finally, we calculate the total induced dipole moment at photon energy $\Omega$ of an ensemble of molecules partially aligned along the direction of the probe field as follows

$$
d(\Omega)=\int_{0}^{\pi} \mathrm{d} \theta \sin \theta A(\theta) \sum_{i} \sqrt{I_{i}(\theta)} a_{e w p, i}(\Omega) d_{r e c, i}(\Omega, \theta)
$$

where $\theta$ is the angle between the molecule and the polarization direction of the probe field, $i$ runs over the relevant ionization channels (ionization from HOMO or HOMO-1), $A(\theta)$ is the axis distribution, $I_{i}$ is the strong-field ionization rate, $a_{e w p, i}$ is the complex photoelectron wave packet and $d_{r e c, i}$ are the complex recombination matrix elements described above.

We illustrate these theoretical results by first restricting the calculations to the HOMO channel and using $a_{\text {ewp }}(\Omega)=1$ with a typical axis distribution $\left(\left\langle\cos ^{2} \theta\right\rangle=0.60\right.$, taken from Ref. [42]). Figure 2d shows the obtained squared amplitude and phase with photorecombination restricted to the $\mathrm{N}$-side $\left(\alpha=+90^{\circ}\right.$ to $\left.+270^{\circ}\right)$ and Fig. 2e for recombination from the O-side $\left(\alpha=-90^{\circ}\right.$ to $\left.+90^{\circ}\right)$. Photorecombination from the O-side leads to two distinct minima and associated phase variations at 29 and $59 \mathrm{eV}$, respectively, whereas recombination from the $\mathrm{N}$-side is characterized by a structureless spectral amplitude and phase. These results differ markedly from the plane-wave approximation of the continuum that yields photorecombination matrix elements from the two sides that are simply complex conjugates of each other [19-21, 43]. The substantial differences between recollision from the two sides are thus the result of the asymmetric diffraction of the photoelectron wave packet in the molecular potential prior to recombination. Minima are thus only found to occur in the emitted spectra when the electron wave packet first encounters the oxygen atom rather than the nitrogen atom, revealing an interesting sensitivity of photorecombination to molecular asymmetries.

The results of calculations using Eq. (1) with both channels and the experimental conditions used in Fig. 1 are shown in Fig. 3. For all wavelengths and intensities the minimum positions and their intensity dependence are well reproduced. The analysis of the calculations, that is presented in detail elsewhere [30], shows that the minimum position is found to 
depend most strongly on the intensity when probe pulses with short wavelengths are used. Under such conditions, the emission from HOMO and HOMO-1 is comparable over a range of harmonics in the cut-off region, which leads to the large shift observed at $1.17 \mu \mathrm{m}$. With increasing wavelength and similar intensities, the cut-off increases and becomes sharper in the energy domain such that the minima appear in the plateau region of the spectrum, where the contributions from lower orbitals are smaller. This leads to the observed decrease of the intensity dependence observed at 1.36 and $1.46 \mu \mathrm{m}$. We have thus shown that our measurements isolate a structural signature of the $\mathrm{N}_{2} \mathrm{O}$ molecule in the spectra measured at $1.46 \mu \mathrm{m}$ and intensities above $0.85 \times 10^{14} \mathrm{~W} / \mathrm{cm}^{2}$.

We now study the origin of this minimum by comparing its position to that observed in $\mathrm{CO}_{2}$. High-harmonic spectra recorded in aligned $\mathrm{CO}_{2}$ and $\mathrm{N}_{2} \mathrm{O}$ molecules under identical experimental conditions and a wavelength of $1.46 \mu \mathrm{m}$ are shown in Fig. 4a. The $\mathrm{N}_{2} \mathrm{O}$ spectrum in Fig. 4a is the same as that shown in Fig. 1c using the highest probe intensity $\left(0.93 \times 10^{14} \mathrm{~W} / \mathrm{cm}^{2}\right.$ determined by comparison with calculations $)$. Before comparing the minimum positions directly, we must take into account the different polarizabilities [44] and therefore slightly different axis distributions of the molecules under identical experimental conditions. Calculations of the rotational dynamics of the two molecules using the experimental parameters $\left(120 \mathrm{fs}\right.$ pulse duration, $T_{\text {rot }}=40 \mathrm{~K}$, intensity in the range of $2 \times 10^{13}$ $\mathrm{W} / \mathrm{cm}^{2}$ ) show that $\mathrm{N}_{2} \mathrm{O}$ reaches a consistently higher degree of $\left\langle\cos ^{2} \theta\right\rangle$ by $0.04-0.05$ compared to $\mathrm{CO}_{2}$ (Fig. 4b). We thus determine the experimental degrees of alignment in $\mathrm{N}_{2} \mathrm{O}$ and $\mathrm{CO}_{2}$ separately by comparing the observed minimum positions to calculations (Fig. 4c). We obtain a degree of axis alignment of $\left\langle\cos ^{2} \theta\right\rangle=0.59$ and 0.64 for $\mathrm{CO}_{2}$ and $\mathrm{N}_{2} \mathrm{O}$, respectively, in excellent agreement with the difference expected from simulations. With this knowledge, we can now predict the spectra of $\mathrm{CO}_{2}$ and $\mathrm{N}_{2} \mathrm{O}$ for identical axis distributions (see Fig. 4d). We find that the minimum positions differ by $10 \mathrm{eV}$.

We now analyze this remarkable finding. The overall length of the molecules is nearly identical with $\mathrm{R}_{g e o}=2.312 \pm 0.003 \AA$ in $\mathrm{N}_{2} \mathrm{O}$ and $2.324 \pm 0.003 \AA$ in $\mathrm{CO}_{2}$ (see Fig. 4e). Thus, the traditional two-center model which has been applied to $\mathrm{CO}_{2}$ and $\mathrm{N}_{2} \mathrm{O}$ e.g. in Refs. $[11,17]$ cannot explain this difference. However, our model described above quantitatively accounts for this experimental observation in terms of photorecombination to the HOMO. We now look for a physical interpretation of the different minimum positions between $\mathrm{CO}_{2}$ and $\mathrm{N}_{2} \mathrm{O}$ using the two-center interference formula $\left(n \lambda_{d B}=R \cos \theta\right)$ backwards. With $\theta=30^{\circ}$ 
(which is the alignment angle with the largest contribution in the sense that $A(\theta) \sin (\theta) \sqrt{I(\theta)}$ is maximal) and converting the photon energy $(\Omega)$ of the minimum to an electron de-Broglie wavelength $\left(\lambda_{d B}\right)$ using $\Omega=2 \pi^{2} / \lambda_{d B}^{2}$ (in atomic units), we obtain an internuclear separation of $R=2.16 \pm 0.10 \AA$ for $\mathrm{CO}_{2}$, which is in moderate agreement with the separation $R_{\text {geo }}$ of the two $\mathrm{O}$ atoms of $2.32 \AA[22]$ (see Fig. 4e). Applying the same approach to $\mathrm{N}_{2} \mathrm{O}$, results in $R=1.94 \pm 0.10 \AA$. This deviates even more strongly from the overall length of $\mathrm{N}_{2} \mathrm{O}$ of $2.31 \AA$. However, the separation of the centers of gravity of the orbital lobes of the HOMOs, $R_{\text {orb }}$, amounts to $2.25 \pm 0.02 \AA$ for $\mathrm{CO}_{2}$, and $2.15 \pm 0.02 \AA$ for $\mathrm{N}_{2} \mathrm{O}$. Given the simplicity of the model, these numbers are in reasonable agreement with the effective lengths extracted from the experiment. We can therefore attribute the difference in the observed minimum positions to the shorter separation of the orbital lobes in the HOMO of $\mathrm{N}_{2} \mathrm{O}$ and to the deviation of the orbital from a simple two-center model. This result is demonstrated more rigorously in Fig. 4f where the difference in the minima is adequately predicted by the pure HOMO emission channel for both molecules.

In conclusion, we have thus shown the first signatures of ionization from lower orbitals in high-harmonic spectra of $\mathrm{N}_{2} \mathrm{O}$ and the disappearance of this effect with increasing wavelength under otherwise identical conditions. This observation has been rationalized through the position of the observed minimum relative to the high-harmonic cut-off and the associated relative emission strength from different channels, as demonstrated in a full theoretical model. This approach enabled us to experimentally isolate the structural minimum in $\mathrm{N}_{2} \mathrm{O}$ and to compare it with that in $\mathrm{CO}_{2}$. Taking the different axis distributions into account, we then showed that the minimum is located $\sim 10 \mathrm{eV}$ higher in $\mathrm{N}_{2} \mathrm{O}$ than in $\mathrm{CO}_{2}$, although the molecules have nearly identical lengths, which is validated by our ab initio calculations. Using the weakly polar $\mathrm{N}_{2} \mathrm{O}$ molecule, we have thus shown that quantum interference in polar molecules distinguishes electronic structure from nuclear geometry. This insight may have important implications in applying high-harmonic spectroscopy to ultrafast photochemical dynamics [28, 45], suggesting that the technique might discern between electronic and nuclear rearrangements. This would be particularly useful in the case of vibronic coupling which is ubiquitous in the dynamics of polyatomic molecules. 


\section{Acknowledgments}

We gratefully acknowledge funding from the Swiss National Science Foundation (PP00P2_128274), ETH Zürich (ETH-33 10-3 and Postdoctoral Fellowship Program) and the Marie Curie COFUND program.

[1] J. Itatani, J. Levesque, D. Zeidler, H. Niikura, H. Pépin, J. C. Kieffer, P. B. Corkum, and D. M. Villeneuve, Nature 432, 867 (2004).

[2] T. Kanai, S. Minemoto, and H. Sakai, Nature 435, 470 (2005), ISSN 0028-0836, URL http: //dx.doi.org/10.1038/nature03577.

[3] C. Vozzi, F. Calegari, E. Benedetti, J.-P. Caumes, G. Sansone, S. Stagira, M. Nisoli, R. Torres, E. Heesel, N. Kajumba, et al., Phys. Rev. Lett. 95, 153902 (pages 4) (2005), URL http: //link.aps.org/abstract/PRL/v95/e153902.

[4] W. Boutu, S. Haessler, H. Merdji, P. Breger, G. Waters, M. Stankiewicz, L. J. Frasinski, R. Taieb, J. Caillat, A. Maquet, et al., Nature Physics 4, 545 (2008), ISSN 1745-2473, URL http://dx.doi.org/10.1038/nphys964.

[5] X. Zhou, R. Lock, W. Li, N. Wagner, M. M. Murnane, and H. C. Kapteyn, Phys. Rev. Lett. 100, 073902 (2008), URL http://link.aps.org/abstract/PRL/v100/e073902.

[6] O. Smirnova, Y. Mairesse, S. Patchkovskii, N. Dudovich, D. M. Villeneuve, P. B. Corkum, and M. Y. Ivanov, Nature 460, 972 (2009), ISSN 0028-0836, URL http://dx.doi.org/10. 1038/nature08253.

[7] S. Haessler, J. Caillat, W. Boutu, C. Giovanetti-Teixeira, T. Ruchon, T. Auguste, Z. Diveki, P. Breger, A. Maquet, B. Carre, et al., Nat Phys 6, 200 (2010), ISSN 1745-2473, URL http: //dx.doi.org/10.1038/nphys1511.

[8] H. J. Wörner, J. B. Bertrand, P. Hockett, P. B. Corkum, and D. M. Villeneuve, Phys. Rev. Lett. 104, 233904 (2010), URL http://link.aps.org/doi/10.1103/PhysRevLett. 104.233904.

[9] C. Vozzi, M. Negro, F. Calegari, G. Sansone, M. Nisoli, S. De Silvestri, and S. Stagira, Nat Phys 7, 822 (2011), ISSN 1745-2473, URL http://dx.doi.org/10.1038/nphys2029.

[10] K. Kato, S. Minemoto, and H. Sakai, Phys. Rev. A 84, 021403 (2011), URL http://link. 
aps.org/doi/10.1103/PhysRevA.84.021403.

[11] R. M. Lock, S. Ramakrishna, X. Zhou, H. C. Kapteyn, M. M. Murnane, and T. Seideman, Phys. Rev. Lett. 108, 133901 (2012), URL http://link.aps.org/doi/10.1103/ PhysRevLett.108.133901.

[12] G. N. Gibson, R. R. Freeman, and T. J. McIlrath, Phys. Rev. Lett. 67, 1230 (1991)

[13] B. K. McFarland, J. P. Farrell, P. H. Bucksbaum, and M. Gühr, Science 322, 1232 (2008).

[14] H. Akagi, T. Otobe, A. Staudte, A. Shiner, F. Turner, R. Dörner, D. M. Villeneuve, and P. B. Corkum, Science 325, 1364 (2009), URL http://www.sciencemag.org/cgi/content/ abstract/325/5946/1364.

[15] J. P. Farrell, S. Petretti, J. Förster, B. K. McFarland, L. S. Spector, Y. V. Vanne, P. Decleva, P. H. Bucksbaum, A. Saenz, and M. Gühr, Phys. Rev. Lett. 107, 083001 (2011), URL http: //link.aps.org/doi/10.1103/PhysRevLett.107.083001.

[16] R. Torres, T. Siegel, L. Brugnera, I. Procino, J. G. Underwood, C. Altucci, R. Velotta, E. Springate, C. Froud, I. C. E. Turcu, et al., Phys. Rev. A 81, 051802 (2010), URL http://link.aps.org/doi/10.1103/PhysRevA.81.051802.

[17] R. Torres, T. Siegel, L. Brugnera, I. Procino, J. G. Underwood, C. Altucci, R. Velotta, E. Springate, C. Froud, I. C. E. Turcu, et al., Opt. Express 18, 3174 (2010), URL http: //www .opticsexpress.org/abstract.cfm?URI=oe-18-3-3174.

[18] A.-T. Le, R. R. Lucchese, S. Tonzani, T. Morishita, and C. D. Lin, Phys. Rev. A 80, 013401 (2009), URL http://link.aps.org/abstract/PRA/v80/e013401.

[19] X. Zhu, Q. Zhang, W. Hong, P. Lan, and P. Lu, Opt. Express 19, 436 (2011), URL http: //www .opticsexpress .org/abstract. cfm?URI=oe-19-2-436.

[20] B. B. Augstein and C. F. de Morisson Faria, Journal of Physics B: Atomic, Molecular and Optical Physics 44, 055601 (2011), ISSN 0953-4075, URL http://stacks . iop.org/0953-4075/ $44 / i=5 / a=055601$.

[21] A. Etches, M. B. Gaarde, and L. B. Madsen, Phys. Rev. A 84, 023418 (2011), URL http: //link.aps.org/doi/10.1103/PhysRevA.84.023418.

[22] G. Herzberg, Molecular Spectra and Molecular Structure, Volume III, Electronic Spectra and Electronic Structure of Polyatomic Molecules (Krieger Publishing Company, Malabar, 1991), 2nd ed.

[23] M. Lein, N. Hay, R. Velotta, J. P. Marangos, and P. L. Knight, Phys. Rev. Lett. 88, 183903 
(2002).

[24] J. Itatani, D. Zeidler, J. Levesque, M. Spanner, D. M. Villeneuve, and P. B. Corkum, Phys. Rev. Lett. 94, 123902 (2005).

[25] H. J. Wörner, H. Niikura, J. B. Bertrand, P. B. Corkum, and D. M. Villeneuve, Phys. Rev. Lett. 102, 103901 (2009), URL http://link.aps.org/abstract/PRL/v102/e103901.

[26] M. Lein, Phys. Rev. Lett. 94, 053004 (2005).

[27] S. Baker, J. S. Robinson, C. A. Haworth, H. Teng, R. A. Smith, C. C. Chirila, M. Lein, J. W. G. Tisch, and J. P. Marangos, Science 312, 424 (2006).

[28] H. J. Wörner, J. B. Bertrand, B. Fabre, J. Higuet, H. Ruf, A. Dubrouil, S. Patchkovskii, M. Spanner, Y. Mairesse, V. Blanchet, et al., Science 334, 208 (2011).

[29] P. M. Kraus, A. Rupenyan, and H. J. Wörner, Phys. Rev. Lett. 109, 233903 (2012).

[30] A. Rupenyan, P. M. Kraus, J. Schneider, and H. J. Wörner, Phys. Rev. A, accepted (2012).

[31] K. Kimura, S. Katsumata, Y. Achiba, T. Yamazaki, and S. Iwata, Handbook of HeI photoelectron spectra (Japan Scientific Societies Press, Tokyo, 1981).

[32] J. Muth-Böhm, A. Becker, and F. H. M. Faisal, Phys. Rev. Lett. 85, 2280 (2000).

[33] A. Jaron-Becker, A. Becker, and F. H. M. Faisal, Journal of Physics B: Atomic, Molecular and Optical Physics 36, L375 (2003), URL http://stacks .iop.org/0953-4075/36/i=21/a=L01.

[34] H. Li, D. Ray, S. De, I. Znakovskaya, W. Cao, G. Laurent, Z. Wang, M. F. Kling, A. T. Le, and C. L. Cocke, Phys. Rev. A, 84, 043429 (2011).

[35] D. Dimitrovski, C. P. J. Martiny, and L. B. Madsen, Phys. Rev. A, 82, 053404 (2010).

[36] L. Holmegaard, J. L. Hansen, L. Kalhoj, S. Louise Kragh, H. Stapelfeldt, F. Filsinger, J. Kupper, G. Meijer, D. Dimitrovski, M. Abu-samha, et al., Nat Phys 6, 428 (2010), ISSN 1745-2473, URL http://dx.doi.org/10.1038/nphys1666.

[37] V. S. Yakovlev, M. Ivanov, and F. Krausz, Opt. Express 15, 15351 (2007), URL http://www. opticsexpress.org/abstract.cfm?URI=oe-15-23-15351.

[38] T. Kanai, E. J. Takahashi, Y. Nabekawa, and K. Midorikawa, Phys. Rev. Lett. 98, 153904 (2007).

[39] H. J. Wörner, J. B. Bertrand, P. B. Corkum, and D. M. Villeneuve, Phys. Rev. Lett. 105, 103002 (2010), URL http://link.aps.org/doi/10.1103/PhysRevLett.105.103002.

[40] A. P. P. Natalense and R. R. Lucchese, J. Chem. Phys. 111, 5344 (1999), URL http://link. aip.org/link/?JCP/111/5344/1. 
[41] F. A. Gianturco, R. R. Lucchese, and N. Sanna, J. Chem. Phys. 100, 6464 (1994), URL http://link.aip.org/link/?JCP/100/6464/1.

[42] A. Rupenyan, J. B. Bertrand, D. M. Villeneuve, and H. J. Wörner, Phys. Rev. Lett. 108, 033903 (2012), URL http://link.aps.org/doi/10.1103/PhysRevLett.108.033903.

[43] A. Etches and L. B. Madsen, Journal of Physics B: Atomic, Molecular and Optical Physics 43, 155602 (2010), ISSN 0953-4075, URL http://stacks.iop.org/0953-4075/43/i=15/a= 155602.

[44] D. Jonsson, P. Norman, H. Agren, A. Rizzo, S. Coriani, and K. Ruud, J. Chem. Phys. 114, 8372 (2001), URL http://dx.doi.org/10.1063/1.1364678.

[45] H. J. Wörner, J. B. Bertrand, D. V. Kartashov, P. B. Corkum, and D. M. Villeneuve, Nature 466, 604 (2010), ISSN 0028-0836, URL http://dx.doi.org/10.1038/nature09185. 

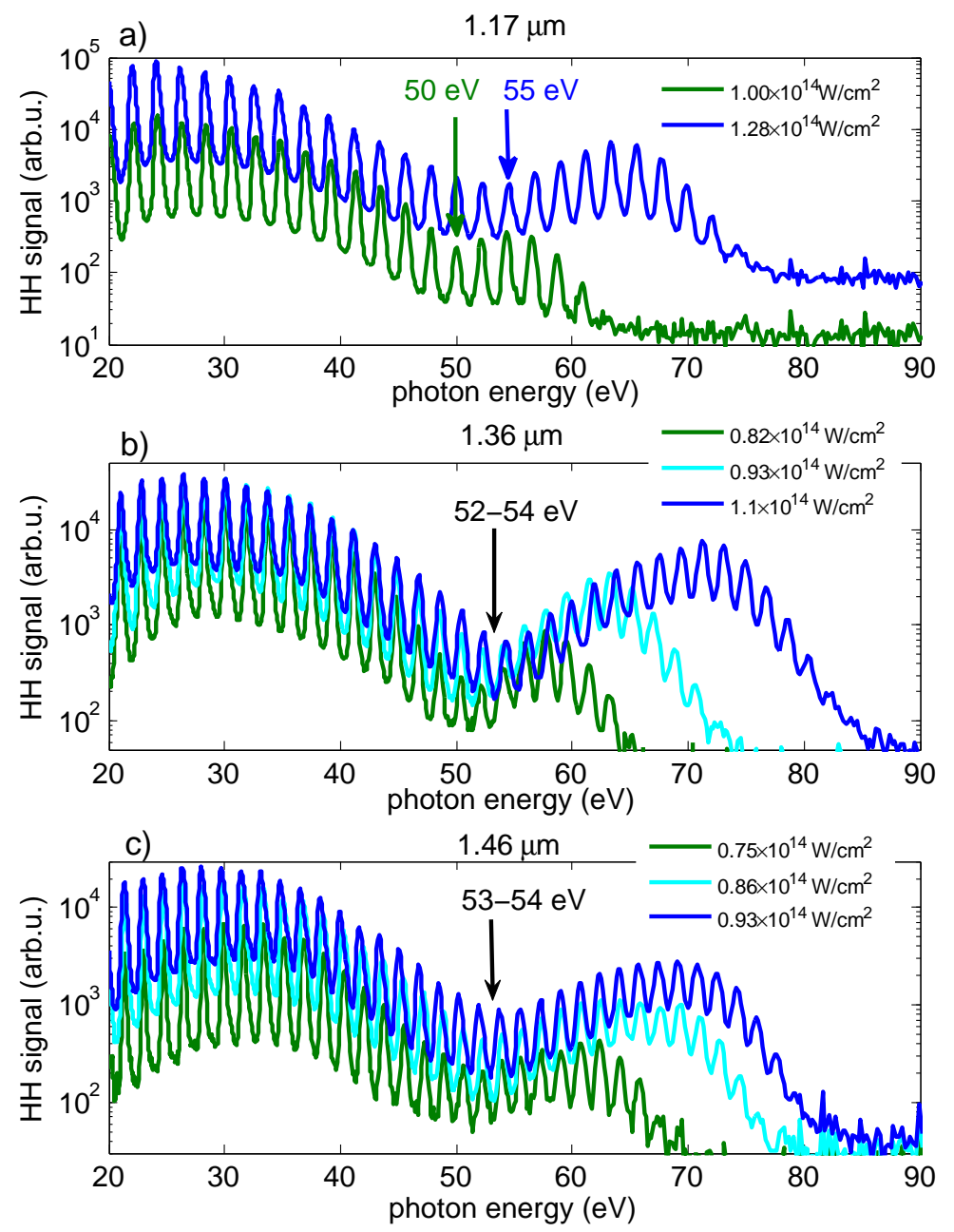

FIG. 1: High-harmonic spectra generated in transiently-aligned $\mathrm{N}_{2} \mathrm{O}$ molecules with 40-50 fs laser pulses with central wavelengths $1.17 \mu \mathrm{m}(\mathrm{a}), 1.36 \mu \mathrm{m}(\mathrm{b})$ and $1.46 \mu \mathrm{m}$ (c) and a range of intensities indicated in the legends. The arrows indicate the positions of the observed minima. 

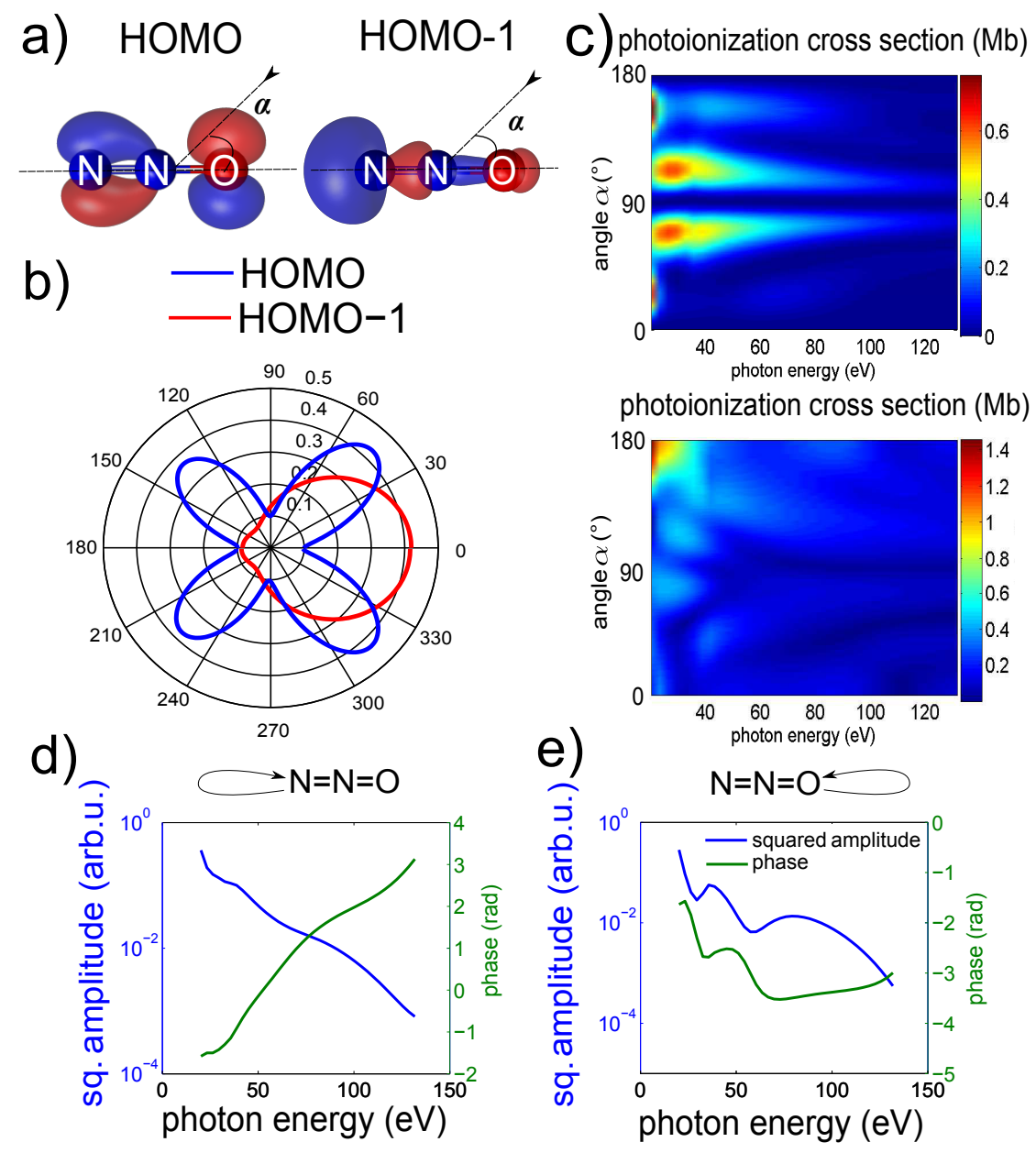

FIG. 2: a) HOMO and HOMO-1 orbitals of $\mathrm{N}_{2} \mathrm{O}$ from a $\mathrm{HF} / /$ cc-pVTZ calculation. b) angular variation of the strong-field ionization rates calculated for an intensity of $1.0 \times 10^{14} \mathrm{~W} / \mathrm{cm}^{2}$. c) photoionization cross sections of HOMO (top panel) and HOMO-1 (bottom panel) as a function of the emitted photon energy and the electron emission angle $\alpha$ in the molecular frame. d) and e) calculated squared amplitude and phase of the induced dipole moment for recombination from a single side of the molecule, using a typical axis distribution characterized by $\left\langle\cos ^{2} \theta\right\rangle=0.60$. 

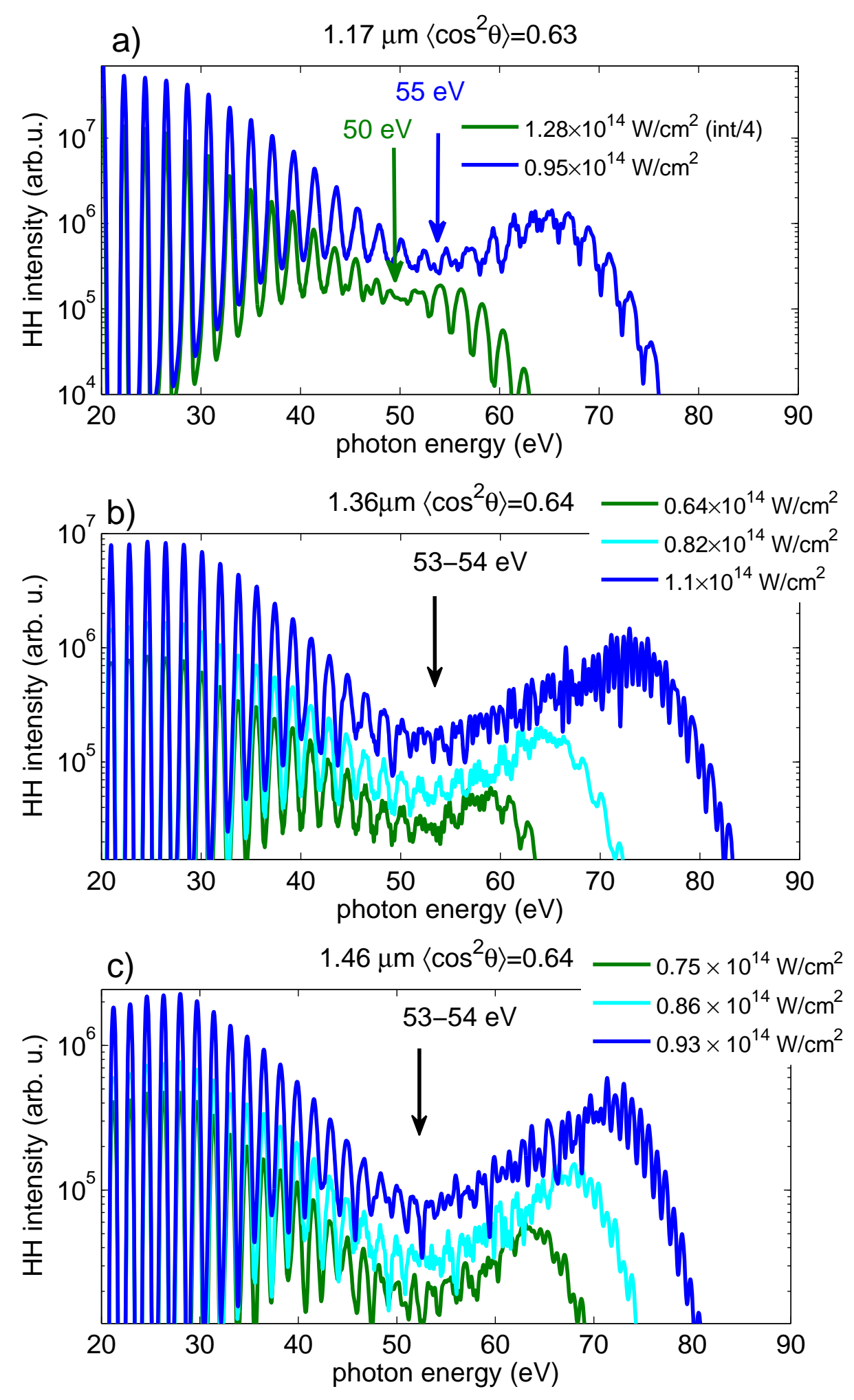

FIG. 3: Calculated high-harmonic spectra including HOMO and HOMO-1 using the quantities shown in Fig. 2 and calculated photoelectron wave packets. Wavelengths, intensities and pulse durations are chosen to match the experimental conditions of Fig. 1. The degree of axis alignment $\left(\left\langle\cos ^{2} \theta\right\rangle\right)$ has been minimally adjusted to reproduce the experimental spectra and is indicated in each panel. 

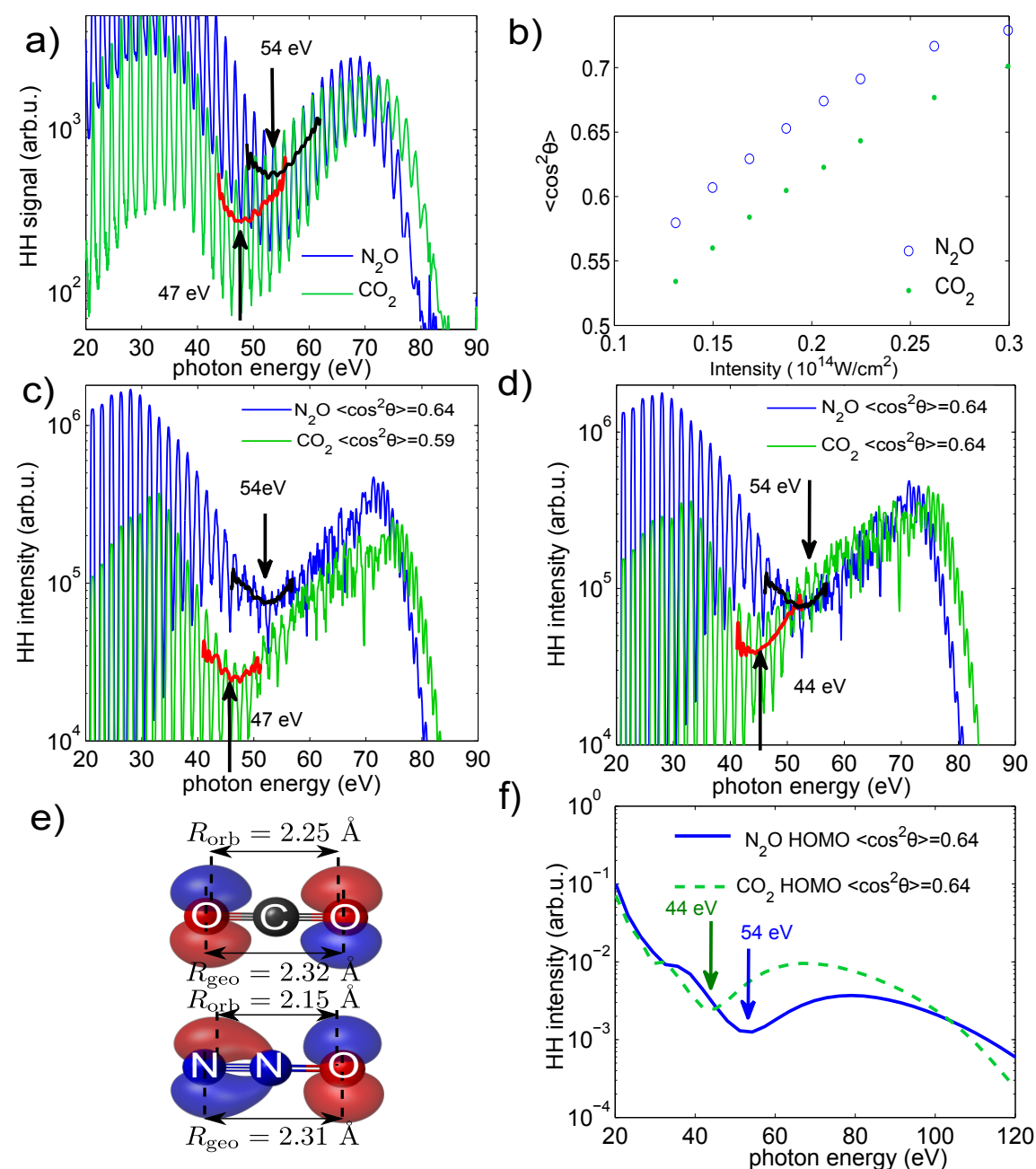

FIG. 4: a) Experimental high-harmonic spectra recorded under identical conditions with a driving wavelength of $1.46 \mu \mathrm{m}$. The positions of the minima are highlighted using smoothed replica of the spectra. b) Calculated maximal degrees of axis alignment for $\mathrm{N}_{2} \mathrm{O}$ and $\mathrm{CO}_{2}$ for $T_{\text {rot }}=40 \mathrm{~K}$ impulsively aligned by a 120 fs pulse. c) Calculated spectra with adjusted degrees of axis alignment given in the legend. d) Calculated spectra using the same axis distribution for both molecules. e) HOMOs of $\mathrm{N}_{2} \mathrm{O}$ and $\mathrm{CO}_{2}$. f) Spectral envelope of the high-harmonic emission from the HOMO channel only, assuming an energy-independent photoelectron wave-packet amplitude $\left(a_{\text {ewp }}(\Omega)=1\right)$. 